\title{
DIAGNOSIS OF LEPROSY
}

\author{
By Dr. V. R. KhanolkaR \\ Director, Indian Cancer Research Centre, Parel, Bombay
}

(Reprinted by courtesy of the Editors of TRIANGLE, the Sandoz Journal of Medical Science)

Leprosy is a chronic infective disease which presents a characteristic and easily recognisable picture in its advanced stages. It is not difficult then to diagnose it, if the possibility of infection with M.leprae is borne in mind. However, it is surprising, even in countries where leprosy is endemic, how frequently the disease is misdiagnosed. In countries where the disease is not commonly seen, leprosy patients are treated sometimes for years, as suffering from nervous disorders. It has also been suggested that as a person declared to be suffering from leprosy unfortunately becomes a marked man in his community, "one should not make a diagnosis of leprosy unless the signs are unequivocal" (COCHRANE ${ }^{1}$ ).

The real difficulty in diagnosis arises when leprosy is in the early stages, not necessarily in terms of the duration of the infection, but in the unfolding of its clinical manifestations. It is, however, important to arrive at a correct diagnosis, because it is during these early stages that the disease is amenable to well-conceived treatment, and most of the deformities and other untoward complications which develop in the wake of its advance can be prevented.

The diagnosis of leprosy in these stages demands an answer to three questions which have to be solved in the following sequence: (a) Is the person concerned suffering from leprosy? (b) If the answer is in the affirmative, it is necessary to ascertain the type of disease with which he is afflicted. Is it a "malignant" or lepromatous type, a "benign" or lepride type, or do the signs and symptoms suggest that the malady belongs to the dimorphous or "intermediate" group? (c) Is the disease progressing and active: is it quiescent or in an arrested phase ?* These questions can only be solved af ter a caref ul clinical, bacteriological, histopathological and immunological study of the patient. It is necessary, however, to point out that it is not often feasible to carry out all the examinations on each and every patient, nor will the technical facilities available to the examining doctor in most places permit them. Under such circumstances a clinician relies on his experience and acumen and often makes a fairly shrewd estimate of the patient's morbid condition.

* At the Manila Conference (1931) it was decided to restrict the application of the term "arrested" to cases which had remained bacteriologically and clinically free from active disease for a period of at least two years; to those cases which had remained free for a period of three months or two years, the term "quiescent" was to be applied. 
The diagnostic investigations a re based on a knowledge of the mode of entry of the infecting organisms and the pathological alterations which follow their proliferation in human tissues. A brief outline of our present-day ideas on this subject is therefore given below.

It has recently been shown that the infecting organisms enter the skin and are taken up by regenerating nerve fibres which are present in the dermis and epidermis, particularly in small, growing children. The leprosy bacilli multiply in the axoplasm of the nerve fibres, enter the body of the ScHWANN cells and often remain dormant in that sheltered location for long periods of time. Under the stimulus of certain supervening changes in the functions of the body, as during adolescence, puberty, pregnancy and the onset of mild maladies, the bacilli begin to proliferate in the nerve fibres and appear in large numbers in the intercalated zones, from which they burst out in the endo and perineural tissues. There, they are taken up by histiocytes which gradually become transformed either into lep ra cells or into epithelioid cells depending upon the immunological response of the host to the presence of leprosy bacilli.

The pathological alterations and the subsequent signs which are observed in the nerves and the skin are dependent upon the distribution of the inflammatory exudate in them (whether as compact aggregates (nodular) broad patches (infiltrating) or as diffuse sheets), and upon the functional impairment of the nerve supply to the cutaneous blood vessels and to the deeper cells of the epidermis. In lepromatous leprosy, the alterations in nerves are probably due to pressure exerted by thickly packed lepra cells in the endo and perineural tissues. The nerve affections in this type of disease develop rather slowly; they are often transient and sometimes reversible. In the tuberculoid type, on the other hand, there is necrosis of the cells of the inflammatory exudate, involving the nerve fibres, the collagen material and the perineural tissues, a s a result of the activity and evolution of the epithelioid cells. The consequent changes manifest themselves in a more lasting or even permanent damage to the affected nerves and secondary changes in the skin supplied by them. The changes in the bigger nerves lead to their thickening, with attendant pain and tenderness, sometimes terminating in the formation of a necrotic mass, or a so-called "nerve abscess", in the tuberculoid type of the disease. The damage to the sensory, reflex, sympathetic and motor nerve fibres manifests itself in characteristic dryness and roughness of the skin, anhydrosis, loss of sensation, wasting and paralysis of certain muscles. The gross signs of these changes are facial paralysis, lagophthalmos, corneal anaesthesia, wrist drop and foot drop. The affected tissues are particularly liable to secondary infection, wasting and atrophy. Spontaneous blisters in the anaesthetic areas, ulceration of the skin, extensive osteoporosis and osteo- 
necrosis of the affected bones are the results of these secondary changes. All these, however, are late manifestations. The earliest changes are those associated with hypopigmentation and certain types of sensory disturbances in the skin. These have been vaguely described as anaesthesias which usually set in, in the following order: alterations in thermal, tactile, pain and pressure sensations.

\section{Clinical examination}

This is the most readily available diagnostic procedure. It can be carried out by a practising physician, and does not entail the use of costly equipment or elaborate techniques. A complete and methodical examination of the whole patient is essential. The entire surface of the body has to be systematically examined in bright daylight, and the sensory performance and alterations in the thickness, feel and sensitivity of certain nerves carefully determined. The examining eye, the palpating hand, a feather or a tuft or cotton-wool, a pin and two glass test tubes with hot and cold water, are the sole requisites. It is, however, a matter of prime importance that the physician should know what to look for in a patient suffering from leprosy. The extension, arrest, or even regression of the disease can only be ascertained if as complete a history as possible is obtained and careful records are maintained of all the clinical observations that have been made at more or less regular, periodic examinations.

The leprosy patient usually sees his physician for one or both of the two following physical signs: (1) numbness or loss of sensation in some part of the body and (2) one or more spots, patches, or even large areas of hypopigmentation of the skin. If at the same time the presence of a large number of acid-fast bacilli can be demonstrated in the skin, the diagnosis of leprosy becomes almost indisputable. Such bacilli, however, are not readily seen in a smear obtained from an incision, and to arrive at a reliable diagnosis other evidence has to be collected. For this purpose one examines the cutaneous nerves arising from the suspected spot, and other superficially placed nerves like (a) the great auricular, (b) the ulnar and (c) the radial. It is possible to differentiate the lepromatous, the tuberculoid and the dimorphous macules from one another on the basis of the physical signs and symptoms presented by the patient. As an illustration the clinical features of the three lesions may now be considered:

Tuberculoid macules. The lesions are few in number and tend to be large and asymmetrical in their distribution. They are commonly found on the face, lateral aspect of the extremities, buttocks and scapulae. The lesions are hypopigmented and show sharply defined edges, clearly demarcated from the adjoining normal skin (Fig. 1). Macules on the extremities and buttocks feel dry to the touch and show anhydrosis. Sometimes they possess a scaly or rough surface, 
and anaesthesia either to light touch or warmth is present. Enlargement of the ulnar or common petoneal is also seen. Sometimes there are areas of anaesthesia on the extremities along the course of the major cutaneous nerves, and in cases of long duration this anaesthesia is fairly pronounced.

Lepromatous macules. The lesions are not confined to the face, buttocks, scapulae etc., but are diffusely scattered over the body in an almost symmetrical manner. Pre-leproma macules are usually small and numerous. Their periphery is hazy in outline and fades imperceptibly into the surrounding normal skin. There is hardly any difference in the texture of the skin over the macules from the normal skin around them (Fig. 2). They appear shiny and there is no anhydrosis.

In diffuse lepromatous leprosy it is difficult to detect the affected area due to the gradual coalescence of several ill-defined macules. In such cases the ear-lobes are usually thickened and shiny, and the eyebrows show loss of hair.

Dimorphous macules. In distribution, in appearance and in size these lesions show certain features of both tuberculoid and lepromatous macular leprosy. Some of the macules have vague edges and show slight loss of tactile and thermal sensation. With the progress of the disease large and small lesions, symmetrically distributed, appear on the body (Fig. 3). The larger macules usually show loss of sensibility and their edges are fairly definite. The majority of these lesions are small, generally with indefinite edges. The texture of the skin of the macules is usually rough and occasionally presents a curious creased or wrinkled appearance.

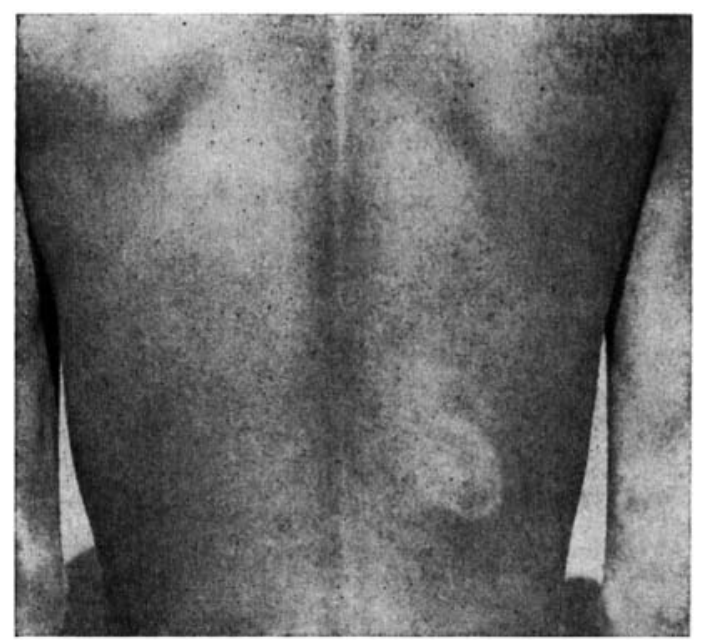

Fig. 1. A typical macule of tuberculoid leprosy. Patient was lepromin positive. 


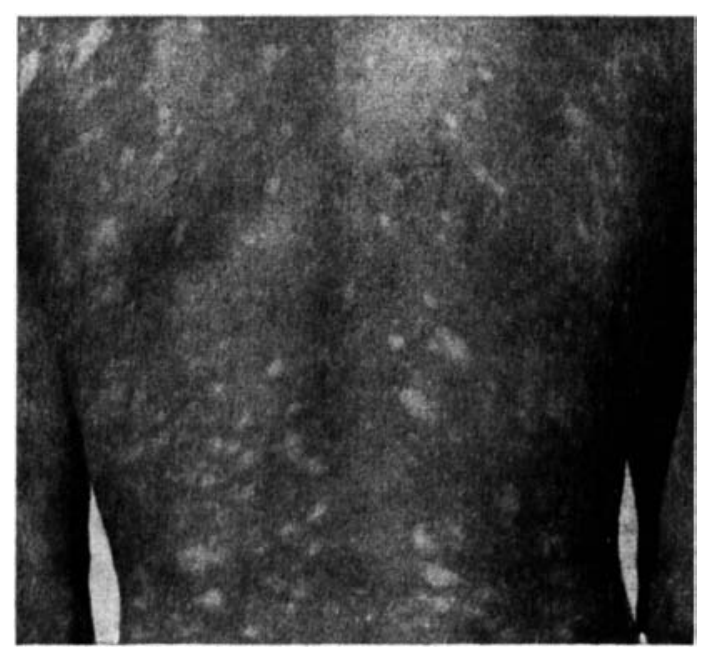

Fig. 2. Multiple macules in lepromatous leprosy. Patient was lepromin negative.

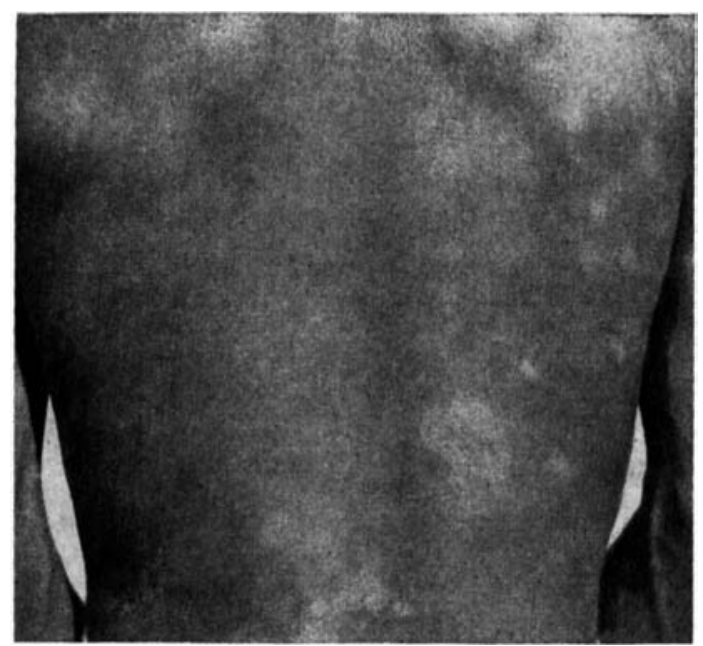

FIG. 3. Macules in dimorphous leprosy, showing features intermediate between the types shown in Figures 1 and 2 . Lepromin was weakly positive.

\section{Bacteriological examination}

The bacteriological examination in leprosy has not progressed beyond a relatively simple procedure, because the causative organism cannot be cultivated on artificial nutrient media, and the disease 
cannot be transmitted to laboratory animals. Therefore the examination involves a microscopic study of smears obtained by superficial incision of the skin. The material is taken by one of the following three methods: In the routine or standard technique a small superficial cut is made in the skin near the advancing edge of the suspected lesion. The blood or lymph which exudes is wiped away and the cut surface scraped with the blade of the scalpel. The material collected on the scalpel is transferred to a microscopic slide and a uniformly thick smear is made from it. The slide is fixed and stained by the ZIEHL-NIFL.SEN technique. The dried smear is then carefully examined under a microscope for the presence of acid-fast bacilli and for the type of inflammatory cells. Some leprologists recommend a deeper cut down to the subcutaneous tissue. We prefer a small piece of tissue $4 \times 3 \times 4 \mathrm{~mm}$. in size for the concentration technique. Instead of a scalpel, a $4 \mathrm{~mm}$. diameter punch may be used for the biopsy. Our patients prefer the punch method of biopsy. It should, however, be pointed out that the punch biopsy is not suitable for histological examination, as there is always a certain amount of crushing of the tissue in the process. The tissue is kept for 3-4 hours in one per cent acetic acid solution which partly fixes it and facilitates the detachment of the epidermis. The tissue left after peeling off the epidermis is minced in a pressure mincer with the addition of 3-4 $\mathrm{ml}$. of normal saline. The supernatant fluid is vigorously shaken after 25 drops of petroleum ether/ethyl ether mixture $1: 10$ have been added to float the micro-organisms to the top layer. Smears are prepared from this layer and fixed in CARNOY's fixative, dried and stained for 3-4 minutes in an aqueous solution containing 0.3 per cent auramine, 0.3 per cent phenol and 6 per cent absolute alcohol. The smears are decolourised for 3-4 minutes in 70 per cent alcohol containing 0.5 per cent of hydrochloric acid and 0.5 per cent of sodium chloride. After a brief rinse in water, the smears are dried and examined under a fluorescence microscope using $40 \times$ objective and $10 \times$ eyepiece. If the auramine-stained smears a re kept in ultra-violet light for more than half an hour, the intensity of the fluorescence gradually fades. However, the smears can be restained with auramine solution. The smears can also be stained by the ZIEHL-NIELSEN technique after staining with auramine solution, but the process cannot be reversed. This technique has given us uniformly good results and we have been able to demonstrate acid-fast bacilli from almost all the lesions of active and even quiescent* leprosy².

\section{Histopathological examination}

A piece of tissue is taken from the selected area. The skin is cleaned and infiltrated with local anaesthetic. After about 5 minutes a suture of sterile thread is passed through the superficial layer of

* See p.158 re Manila Conference. 
the corium at one of the extreme ends of the proposed biopsy. The thread is used to steady the piece of tissue to be removed and to avoid the use of forceps which always crush the intended biopsy and often spoil a good portion of it for histological examination. The thread is gently pulled and an elliptical incision in the skin down to the subcutaneous tissue is made with a sharp sterile scalpel. A piece of skin measuring approximately $1.5 \mathrm{~cm}$ long and $0.5 \mathrm{~cm}$ wide is obtained without squeezing the tissue, and is immediately transferred to the fixative.

For examination of the biopsy material with an electron microscope, the fixation, dehydration and impregnation are carried out by different techniques which permit the preparation of ultra-thin sections $25-50 \mu$ thick with an ultra-microtome. This is a complicated process which need not be described here.

In the earliest stages of the disease the microscope does not reveal any abnormal features, though a careful search occasionally reveals the presence of histiocytes with one or two intact or fragmented acid-fast bacilli in their cytoplasm. Sometimes the cytoplasm stains diffusely with the basic dye which does not decolourise with weak acid. These cells have been seen by us only in the early stages of leprosy and have been termed "fuchsinophil" cells. At a slightly later stage a non-specific type of cellular exudate is seen in the microscopic preparations which present more characteristic features of infection with M.leprae. After the disease has progressed further it is possible to identify the tissue reaction and to distinguish a leproma, a tuberculoid or a dimorphous lesion. In the early stages of the lepromatous type of disease it is observed that the axoplasm of some cutaneous nerves has undergone degenerative changes, with leprosy bacilli lying in the disintegrated tissue; the sheath of SCHWANN is collapsed and converted into a ribbon-like band (BüNGNER's band). M.leprae are usually found in large numbers in the nerve fibres and in the endo- and perineural tissues. The bacilli are ingested by the histiocytes which are gradually transformed into lepra (VIRCHOW's) cells. The transformation of the histiocytes is a result of multiplication of the mycobacteria within the cell cytoplasm and their envelopment with electron-opaque lipid material. As a result of the metabolic activity of the bacilli, electron-transparent material begins to show itself in the lipid droplets and forms clear zones to the microorganisms. These clear zones fuse until the whole cytoplasm of the inflammatory cell assumes a foam-like or soap-bubble appearance. In between the lepra cells, groups of mononuclear and plasma cells are often encountered.

In the tuberculoid lesion the dermis is invaded by foci of productive inflammatory exudate mainly comprising mononuclear cells and histiocytes. The most characteristic feature of the inflammatory reaction is the transformation of these histiocytes into epithelioid 


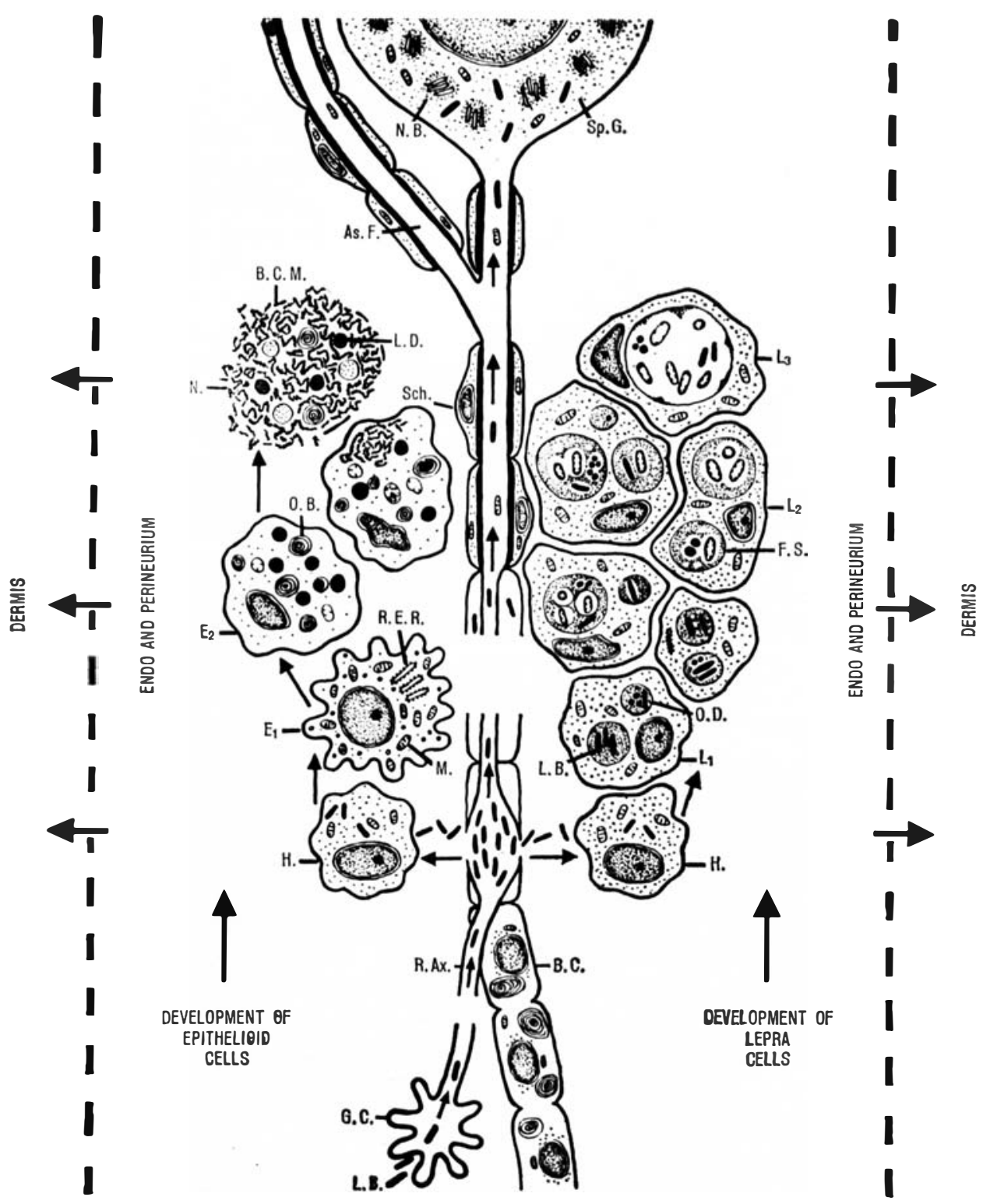

FIG. 4. Diagram of the histological findings in leprosy. As.F.=Ascending fibre; B.C. = BUNGNER's band; B.C.M.=Broken cell membrane; $\dot{E}_{1}=$ Epithelioid cell (1st stage); $\mathrm{E}_{2}=$ Epithelioid cell (2nd stage); F.S.=Foamy structure; G.C.= Growth cone; $\mathrm{H}=$ Histiocyte; $\mathrm{L}_{1}=$ Lepra cell (1st stage); $\mathrm{L}_{2}=$ Lepra cell ( 2 nd stage); $\mathrm{L}_{3}=$ Lepra cell (3rd stage); L.B.= Leprosy bacillus; L.D. = Lipid droplet; M.= Mitochondria; N. = Necrotic mass; N.B. $=$ NISSL body; O.B. $=$ Onion body; O.D.=Opaque droplet; R.Ax.=Regenerating axon; R.E.R.=Roughsurfaced endoplasmic reticulum; Sch.=SCHWANN's cell; Sp.G.=Spinal ganglion cell. 


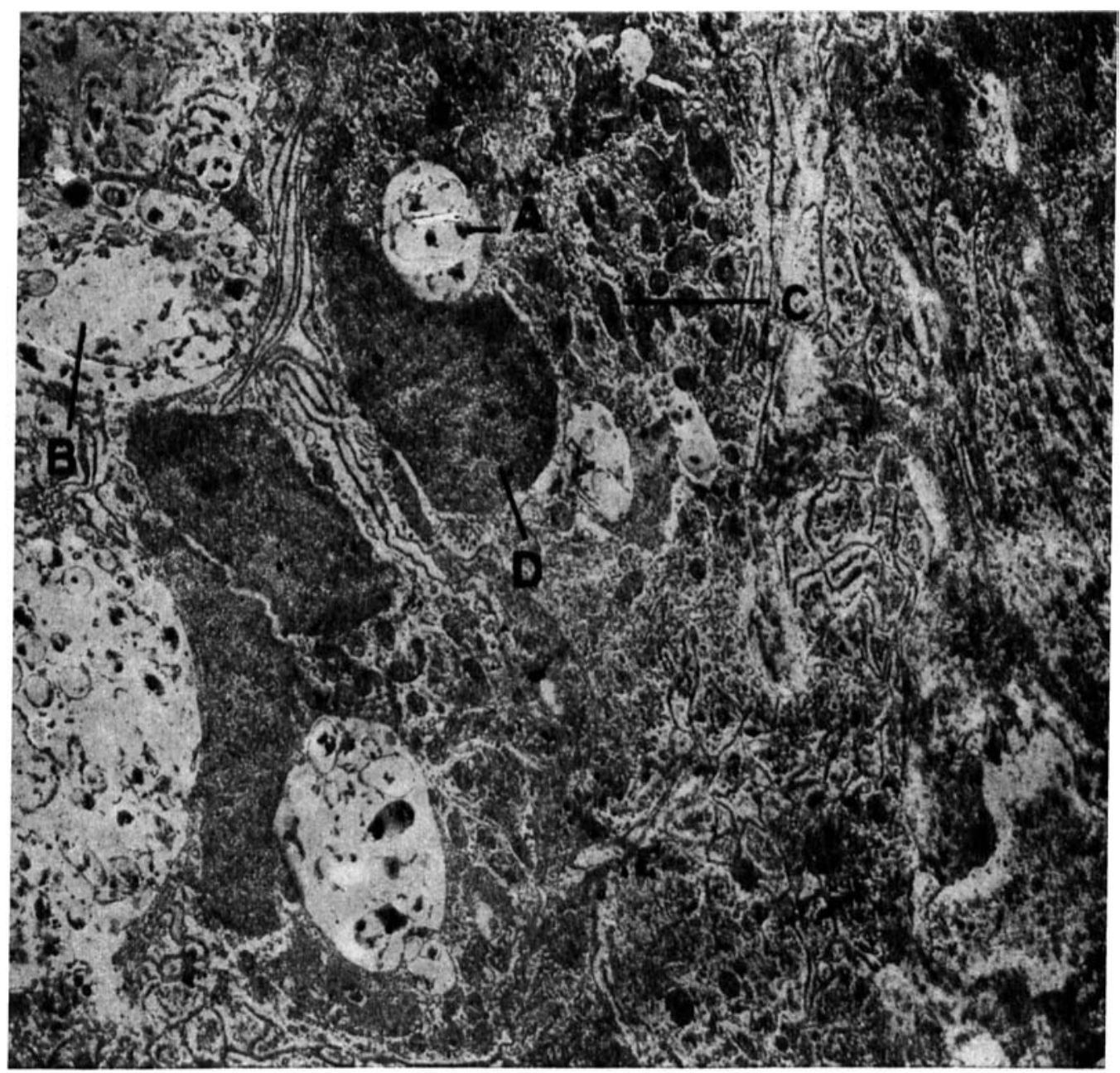

FIG. 6. Advanced foamy cells in a lepromatous great auricular nerve. In the cytoplasm of lepra cells, many degenerated leprosy bacilli are seen embedded in oval electron-transparent zones. The cell wall of the degenerated bacilli is swollen and the bacillary cytoplasm is fragmented. Magnification: 10,000 x. A: Degenerated leprosy bacilli. B: Electron-transparent zone. C: Mitochondria. D: Nucleus. (Electron micrograph).

bacilli or other cells or tissue which come within the range of their activity. The collagen fibres and nerve twigs which are involved in this process undergo degenerative changes and dissolution. The nerve fibres show WALLERIAN degeneration in the early stages and, later, necrosis, when they are incorporated in the substance of the epithelioid tubercle formation. These lesions are characterised by a scarcity of the mycobacteria in the inflammatory tissue (Fig. 4).

The dimorphous lesion presents mixed features of the above two 


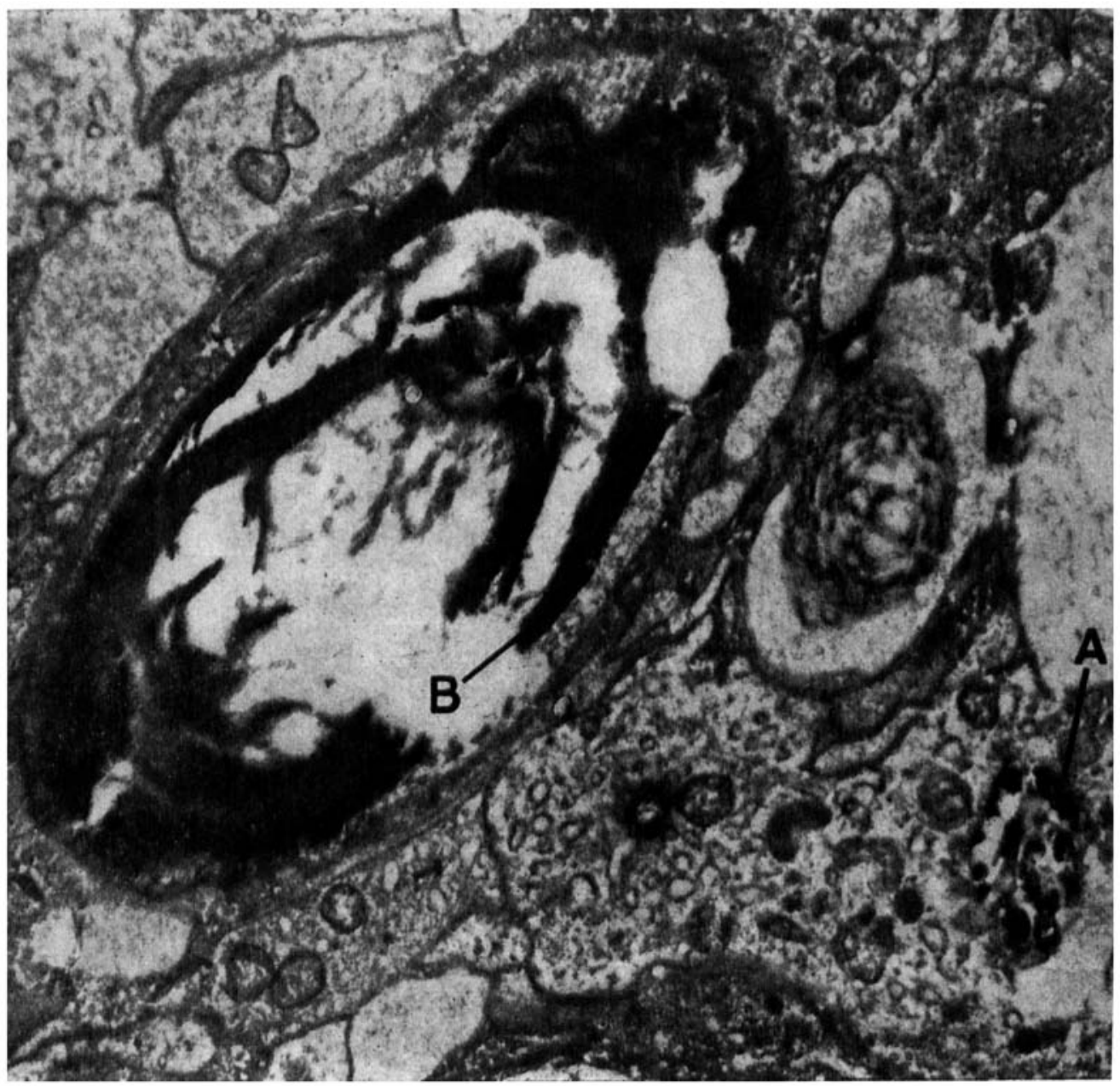

FiG. 5. Lepromatous lesion in a great auricular nerve. A group of leprosy bacilli is seen in a iepra cell of the endoneural space. These bacilli have not yet degenerated and have a more or less homogenous bacillary cytoplasm and nuclear apparatus. Various stages of a degeneration of the myelin sheath are observed. Magnification: 28,000 x. A : Leprosy bacilli. B: Degenerating myelin sheath. (Electron micrograph).

cells and occasional giant cells round a core of fine nerve twigs. These cells are rather large with faintly acidophilic and finely granular cytoplasm. Under the electron microscope the ultra-thin section shows that the cytoplasm contains smooth-surfaced endoplasmic reticulum and evenly scattered lipoid dust comprising weakly osmiophilic droplets. The epithelioid cells are fragile and the cell wall often cracks, leading to a spilling of the cytoplasmic contents into the intercellular tissue. The epithelioid cells engulf and rapidly destroy 
types in the same or different lesions in the same person; it is an intermediate response to the infection of the host tissues with leprosy bacilli.

\section{Immunological examination}

Even the most refined techniques available at the present time fail to reveal the presence of antibodies to M.leprae in the sera of patients. Further the uterine horns of guinea-pigs which have been sensitised to M.leprae do not contract when "shocked" with a small dose of mycobacterial antigen. Therefore it can be stated that our present techniques are incapable of detecting antibodies to M.leprae in leprosy patients and in immunised experimental animals. A technique which has often been used is a skin reaction to lepromin, a product prepared from lepromatous nodules. The reaction is positive in tuberculoid patients and negative in lepromatous patients. As pointed out by FELDMAN ${ }^{3}$ "there is much about the lepromin reaction that is nebulous and purely presumptive. Until more definite information is available, the exact situation pertaining to the capability to react to lepromin must remain the realm of speculation".

\section{References}

1. Cochrane, R. G., "Leprosy in Theory and Practice", p.143, Wright, Bristol 1959.

2. Nerurkar, R. V., Khanolkar, V. R., Indian J. Med. Res. 1956, 44, 397.

3. Feldman, W. H., Karlson, A. G., Grindlay, J. H., Ann. New York Acad. Sc. 1951, 54, 53. 